\title{
Comparing synthesis routes to nano-crystalline zeolite ZSM-5
}

\author{
Gilles Reding, Torsten Mäurer, Bettina Kraushaar-Czarnetzki * \\ Institute of Chemical Process Engineering CVT, University of Karlsruhe (TH), D-76128 Karlsruhe, Germany \\ Received 28 May 2002; received in revised form 4 September 2002; accepted 11 September 2002
}

\begin{abstract}
Aiming at the identification of a suitable synthesis route to zeolite ZSM-5 with acidic properties and crystal diameters of about $100 \mathrm{~nm}$ or less, we have evaluated four different preparation methods. Three of these have been adopted from the literature, and one was developed by ourselves. The products, all of which synthesised from mixtures with a fixed $\mathrm{Si} / \mathrm{Al}$ ratio of 60 , were inspected by means of X-ray diffraction, scanning electron microscopy, laser-Doppler anemometry and temperature-programmed desorption of ammonia. Two methods turned out to be successful and well reproducible. The first one comprises hydrothermal crystallisation from clear solutions under autogenous pressure and has been described previously by van Grieken et al. The other approach is based on the use of colloidal silicalite-1 seed crystals in an open-vessel crystallisation at atmospheric pressure. The crystal size distribution and the $\mathrm{Si} / \mathrm{Al}$ ratio of the products can be well controlled. The products from both synthesis types could be transferred into the H-form by conventional means without causing collapse of the crystal structure.
\end{abstract}

(c) 2002 Elsevier Science Inc. All rights reserved.

Keywords: MFI; Nano-size; Nano-crystals; Colloidal; Crystallisation

\section{Introduction}

It is well known that the efficiency and selectivity of porous catalyst bodies depends on their characteristic lengths or, more precisely, on the number of active sites at the external surface in relation to the number of sites accessible via the pore system. This holds as well for individual zeolite crystals. However, industrial catalysts are shaped bodies, each of which containing ensembles

\footnotetext{
${ }^{*}$ Corresponding author. Tel.: +49-721-608-3947/4133; fax: +49-721-608-6118.

E-mail address: bettina.kraushaar@ciw.uni-karlsruhe.de (B. Kraushaar-Czarnetzki).
}

of many zeolite crystals bound to a porous matrix material that should provide mechanical strength and, optionally, an additional catalytic function. Any effect of the zeolite crystal size is superimposed by the catalytic properties of the materials involved, and by the mass transport properties of the total catalyst body. On the longer term, we are interested in exploring new techniques for the implementation of zeolite nano-crystals into shaped catalyst bodies such that beneficial crystal size effects can be utilised in technical reactors.

So far, only a few examples have been reported documenting a benefit of small zeolite crystals being effective even in shaped catalyst bodies $[1,2]$. These cases refer to the implementation of nanosized zeolite Beta into extruded hydrocracking 
catalysts, resulting in a higher bottoms cracking activity due to the higher share of external zeolite surface. In many other catalytic reactions, an effect of the zeolite crystal size has been evidenced as well, however, with research catalysts consisting of pure zeolite, only.

Conventionally manufactured zeolites, in particular, of structure types BEA and MFI may contain nano-sized crystals. These are typically lumped together in agglomerates or even intergrowths which resist to a complete breakdown to single crystals. Thanks to the pioneering work by Verduijn [3-6] and by Schoeman and coworkers [7-9], it is also possible to prepare zeolites consisting of single crystals with diameters around 100 $\mathrm{nm}$ or less. These syntheses produce colloidal sols of zeolites in uniform crystals sizes and have been developed for the preparation of zeolite films and membranes. We have shown recently that it is possible to control the agglomeration and peptisation of such colloidal zeolites in aqueous media [10]. This is a prerequisite for their efficient solidliquid separation [11,12], and for the manufacturing of catalysts containing small zeolite crystals.

The preparation methods for colloidal zeolites as described by Verduijn and by Schoeman and Sterte are identical to a large extent, and they are applicable to the structure types FAU, LTA, SOD [7], MFI [3,4,7-9], MEL [3,4], OFF [4,5], LTL [4,6] and BEA [4]. The crystallisation is performed from clear solutions in open vessels at low temperature and ambient pressure. For the synthesis of nanocrystalline ZSM-5 (MFI structure), new methods have been reported recently. Van Grieken et al. prepared ZSM-5 under hydrothermal conditions at autogenous pressure from clear supersaturated synthesis mixtures [13]. A "confined space synthesis" of ZSM-5 within the mesopores of impregnated carbon black has been reported by Jacobsen et al. [14].

In this contribution, we compare the different routes known so far to produce nano-crystals of ZSM-5. We have evaluated the synthesis procedures mentioned above, and we will also present a new synthesis method. Emphasis is given to the control of the size distribution and morphology of the crystals, and to the possibility of producing acidic material by incorporation of framework aluminium.

\section{Experimental}

\subsection{Zeolite preparation}

In all syntheses described below, it was our aim to produce ZSM-5 containing framework aluminium in a fixed amount equivalent to an atomic $\mathrm{Si} / \mathrm{Al}$ ratio of 60 . Van Grieken et al. [13] and Jacobsen et al. [14] have provided informative descriptions of their synthesis methods which do not require supplementary instructions. Therefore, the description of these procedures will not be repeated here. Rather, those details will be mentioned which were deviating or specific in our experiments. The syntheses after Verduijn have been published in several patents, and the possibility of synthesising ZSM-5 with aluminium is claimed. However, the examples given there refer exclusively to aluminium-free silicalite- 1 . We have chosen example 1 in Ref. [4] as a basis for our experiments, both in the presence and in the absence of an aluminium source.

Synthesis after Verduijn [4]. Synthesis mixtures of the molar composition $\left(9.12(\mathrm{TPA})_{2} \mathrm{O}: 60 \mathrm{SiO}_{2}\right.$ : $0.5 \mathrm{Al}_{2} \mathrm{O}_{3}: 936 \mathrm{H}_{2} \mathrm{O}$ ) expressed in terms of oxide ratios were prepared by adding successively aluminium sulphate $\left(\mathrm{Al}_{2}\left(\mathrm{SO}_{4}\right)_{3} \cdot 18 \mathrm{H}_{2} \mathrm{O}\right.$, Merck) and silica (Grace) to boiling solutions of $20 \mathrm{wt} . \%$ tetrapropyl-ammonium hydroxide in water (Fluka). After 10 min of stirring under reflux, clear homogenous solutions were obtained. These were cooled down to room temperature, and mass losses due to evaporation were compensated by addition of deionised water. The clear solutions were filled into flasks which were equipped with reflux condensers and mounted into oil baths. Crystallisation took place under atmospheric pressure and at static conditions. Preferably, the temperature was adjusted to $353 \mathrm{~K}$. Products were analysed after different crystallisation times. The products were recovered by centrifugation (at $5000 \mathrm{~min}^{-1}$ ) and repeatedly re-suspended in de-ionised water until the sol exhibited a $\mathrm{pH}$-value of 9-10. When appropriate, the concentrated sol was dried overnight at $393 \mathrm{~K}$.

Synthesis after van Grieken et al. [13]. The procedure was followed as described, using aluminium sulphate $\left(\mathrm{Al}_{2}\left(\mathrm{SO}_{4}\right)_{3} \cdot 18 \mathrm{H}_{2} \mathrm{O}\right.$, Merck $)$, 
tetrapropyl-ammonium hydroxide (20 wt.\% in water, Fluka), and tetraethylorthosilicate (Sivento) to produce final synthesis solutions of the composition (10.7 (TPA) $\left.)_{2} \mathrm{O}: 60 \mathrm{SiO}_{2}: 0.5 \mathrm{Al}_{2} \mathrm{O}_{3}\right)$. Crystallisation was interrupted after $48 \mathrm{~h}$, and product recovery was performed as described above.

Confined space synthesis after Jacobsen et al. [14]. The procedure was followed as described, using aluminium sulphate $\left(\mathrm{Al}_{2}\left(\mathrm{SO}_{4}\right)_{3} \cdot 18 \mathrm{H}_{2} \mathrm{O}\right.$, Merck), tetrapropylammonium hydroxide (20 wt.\% in water, Fluka) and tetraethylortho-silicate (Sivento). Printex L6 (Degussa) exhibiting a pore volume of $1.2 \mathrm{ml} / \mathrm{g}$ was employed as a carbon black material. The synthesis mixture in the pores of the carbon black exhibited the composition (10.8 (TPA) ${ }_{2} \mathrm{O}: 60 \mathrm{SiO}_{2}: 0.5 \mathrm{Al}_{2} \mathrm{O}_{3}: 468 \mathrm{H}_{2} \mathrm{O}: 240 \mathrm{EtOH}$ ).

Atmospheric crystallisation with silicalite-1 seeds. This synthesis method comprises two crystallisation steps. In the first, colloidal silicalite-1 is prepared after Verduijn as described above, however, without addition of an aluminium source. After crystallisation for $72 \mathrm{~h}$ at $353 \mathrm{~K}$ under atmospheric pressure, the silicalite-1 nano-particles were separated from the mother liquor by centrifugation and decantation. Repeatedly, they were redispersed in de-ionised water using an ultrasonic bath, and separated again until a concentrated colloidal suspension was obtained.

In the second step, new synthesis mixtures were prepared by adding aluminium sulphate $\left(\mathrm{Al}_{2}\left(\mathrm{SO}_{4}\right)_{3} \cdot 18 \mathrm{H}_{2} \mathrm{O}\right.$, Merck $)$ and silica $\left(\mathrm{SiO}_{2}\right.$, Grace) to boiling solutions of tetrapropylammonium hydroxide in water (20 wt.\%, Fluka), $10 \mathrm{~min}$ stirring under reflux and cooling down. The clear, homogeneous solutions were then seeded with the colloidal silicalite-1. The composition of the resulting synthesis mixtures expressed in terms of oxide ratios was $\left(9.12\right.$ (TPA) ${ }_{2} \mathrm{O}: 60 \mathrm{SiO}_{2}^{*}: 0.5 \mathrm{Al}_{2} \mathrm{O}_{3}$ : $936 \mathrm{H}_{2} \mathrm{O}$ ). In this formula, the amount of template refers only to the TPAOH added with the solution, whereas template occluded in the silicalite- 1 crystals has not been taken into account. The term $\left(60 \mathrm{SiO}_{2}^{*}\right)$, in contrast, represents the sum of silica contained in the seed crystals and in the silica which was dissolved to produce the clear solution:

$$
\left[\left(60 \mathrm{SiO}_{2}^{*}\right)=\left(a \mathrm{SiO}_{2}\right)_{\text {ex solution }}+\left(b \mathrm{SiO}_{2}\right)_{\text {ex silicalite }}\right] .
$$

We prepared three mixtures in which silicalite-1 amounted to $10 \mathrm{wt} . \%(a=54$ and $b=6), 23 \mathrm{wt} . \%$ $(a=46$ and $b=144)$ and 33 wt. $\%(a=40$ and $b=20$ ), respectively, of the total silica content. After crystallisation for varying times at $353 \mathrm{~K}$ under atmospheric pressure, the products were separated by centrifugation, washed and dried as described before.

Calcination and ion exchange. To produce the acidic $\mathrm{H}$-form, products were calcined in air to remove the template, ion exchanged and calcined again. All calcinations were carried out with very thin layers of the zeolite at $823 \mathrm{~K}$ for $1 \mathrm{~h}$ after heating at a rate of $2 \mathrm{~K} / \mathrm{min}$. Samples were ion exchanged three times in a large excess of aqueous $1.5 \mathrm{M}$ solutions of $\mathrm{NH}_{4} \mathrm{NO}_{3}$ at $323 \mathrm{~K}$.

\subsection{Characterisation}

X-ray diffraction (XRD) patterns were collected with a Siemens D500 diffractometer using $\mathrm{Cu} \mathrm{K} \alpha$ radiation. The morphology and the size of the crystals was examined by means of scanning electron microscopy (Hitachi S-4500). The particle size distributions in sols containing $1 \mathrm{~g} / \mathrm{l}$ of template-containing zeolite were measured by means of laser-Doppler anemometry (Master Sizer, Malvern). To ensure a high dispersion of the crystals in these measurements, the sols were exposed to an ultrasonic treatment, and the $\mathrm{pH}$ was adjusted to 9-10 to maximise the repulsive electrostatic forces between the crystals [10]. Temperatureprogrammed desorption of ammonia (AutoChem 2910, Micromeritics) was carried out to determine the amount of acid sites relative to reference samples of H-ZSM-5 with known Si/Al ratios. Prior to the measurements, the samples were outgassed by heating in helium at $773 \mathrm{~K}$ for 3 h. At a temperature of $493 \mathrm{~K}$, the samples were contacted with a stream containing 10 vol.\% $\mathrm{NH}_{3}$ in He for $30 \mathrm{~min}$, followed by flushing with pure He for $1 \mathrm{~h}$. After cooling down to $323 \mathrm{~K}$, the temperature was increased up to $873 \mathrm{~K}$ at a heating rate of $10 \mathrm{~K} / \mathrm{min}$ while recording the $\mathrm{NH}_{3}$-desorption with a thermal conductivity detector. 


\section{Results}

\subsection{Crystallisation}

All synthesis methods under study yielded crystalline products of MFI-structure except for the confined space synthesis after Jacobsen et al. In this case, amorphous products were obtained. We did not systematically search for the reasons for this failure. It should be noticed, however, that Jacobsen et al. used aluminium isopropoxide or sodium aluminate as aluminium sources, whereas our experiments were performed with aluminium sulphate. In addition, the carbon black was of different origin. These deviations from the original recipe could have contributed to the problem of reproducibility.

The crystallisation of the synthesis mixture after Verduijn was followed by taking samples at different points in time. In Fig. 1(a)-(d), the corresponding XRD patterns are depicted for crystallisation times of 4, 6, 10 and 12 days, respectively. This sequence indicates that 10 , preferably 12 days are required to obtain a fully crystallised product, when aluminium is present in the synthesis mixture. In the absence of aluminium, the crystallisation proceeds much faster. Fig. 2 shows the XRD diffraction pattern of silicalite-1 after a crystallisation time of $72 \mathrm{~h}$. Such products were used as seeds in the two-stage synthesis.

In Figs. 3-5, diffraction patterns of products obtained from two-stage syntheses at atmospheric pressure are shown. The amount of silicalite-1 seed crystals was varied from 10 wt.\% (Fig. 3) to 23 wt.\% (Fig. 4) and 33 wt.\% (Fig. 5), basis the total silica content in the synthesis mixture. The presence of these seed crystals explains the weak diffraction peaks which are already visible at the start of the crystallisation (bottom lines in Figs. 3-5, indicated by " 0 d"). As compared to the Verduijn synthesis without seeds (see Fig. 1), the crystallisation proceeds considerably faster. This accelerating effect increases with increasing concentration of seeds. Using 33 wt.\% of seeds (Fig. 5), the crystallisation of ZSM-5 at atmospheric pressure is completed after 3 days.

In the synthesis after van Grieken et al., the crystallisation time was fixed $(48 \mathrm{~h})$. A typical

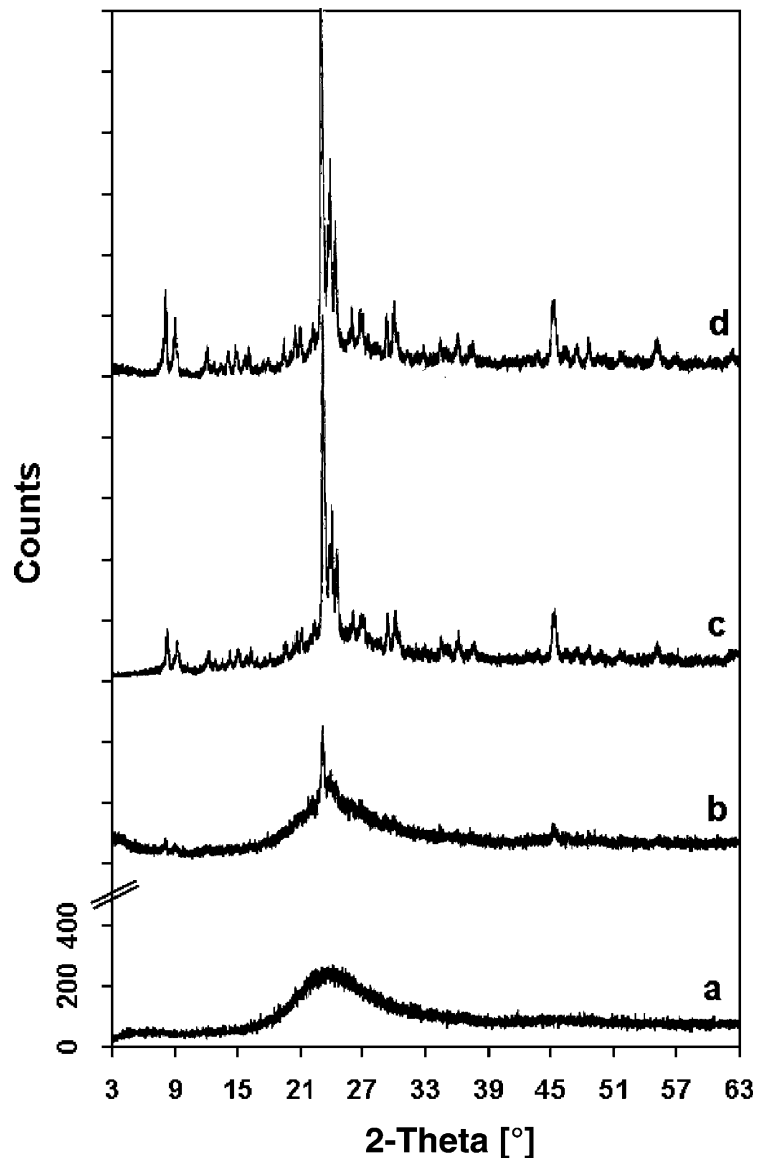

Fig. 1. XRD patterns of ZSM-5 synthesised after Verduijn [4] in the presence of an aluminium source; crystallisation times: 4 days (a), 6 days (b), 10 days (c), and 12 days (d).

XRD pattern of the products is depicted in Fig. 6 . It exhibits a very low background signal and sharp reflections, indicating excellent crystallinity of the sample.

\subsection{Crystal size and morphology}

The presence of aluminium has a strong impact on the particle size distribution of the products obtained from the synthesis after Verduijn. In all experiments, we observed that a broad distribution of particles ranging from $80 \mathrm{~nm}$ up to $800 \mathrm{~nm}$ in diameter is obtained, when aluminium was added to the synthesis solution. As an example, the particle size distribution of an as-synthesised product 


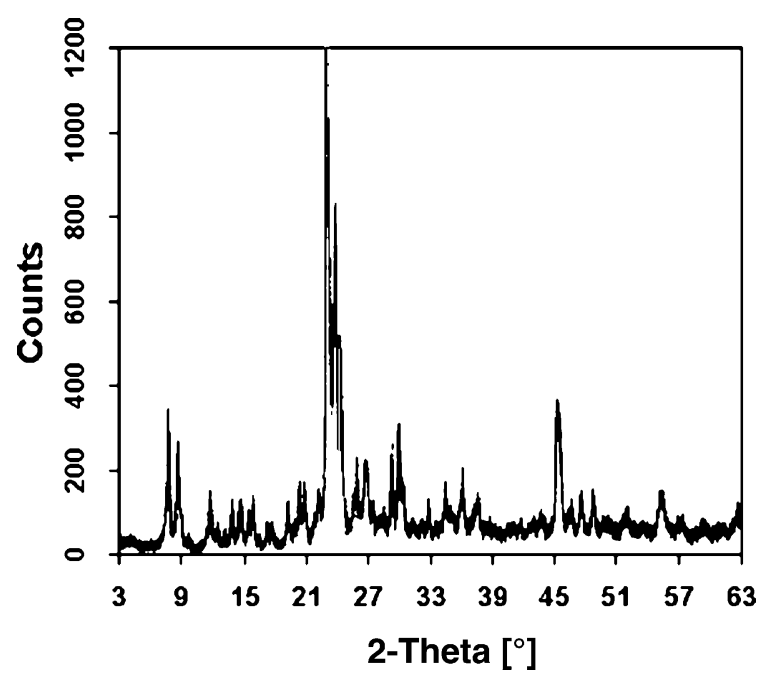

Fig. 2. XRD pattern of silicalite-1 synthesised after Verduijn [4]; crystallisation time: 3 days.

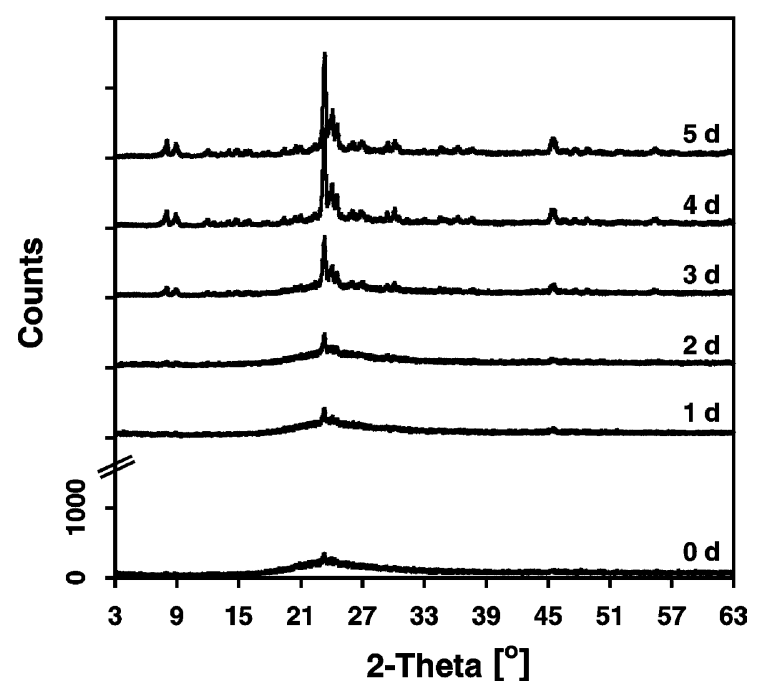

Fig. 3. XRD patterns of ZSM-5 synthesised in two stages with $10 \mathrm{wt} . \%$ silicalite-1 seeds; crystallisation times are indicated on the right side.

in aqueous suspension is shown in Fig. 7, and the corresponding SEM-image of a dried sample is depicted in Fig. 8. The particles have a cauliflowerlike morphology. We assume that these particles are intergrowths formed from nano-sized primary crystals. Our numerous attempts to produce par-

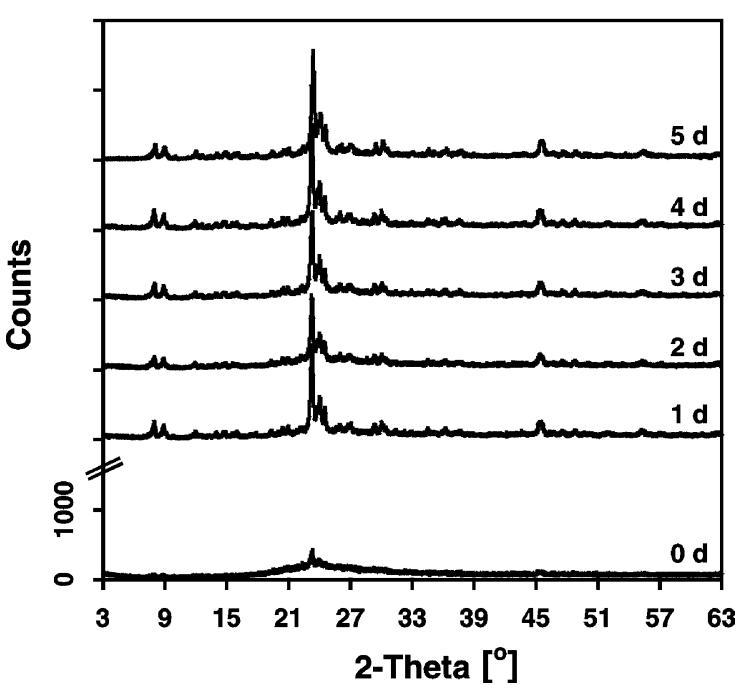

Fig. 4. XRD patterns of ZSM-5 synthesised in two stages with 23 wt. $\%$ silicalite- 1 seeds; crystallisation times are indicated on the right side.

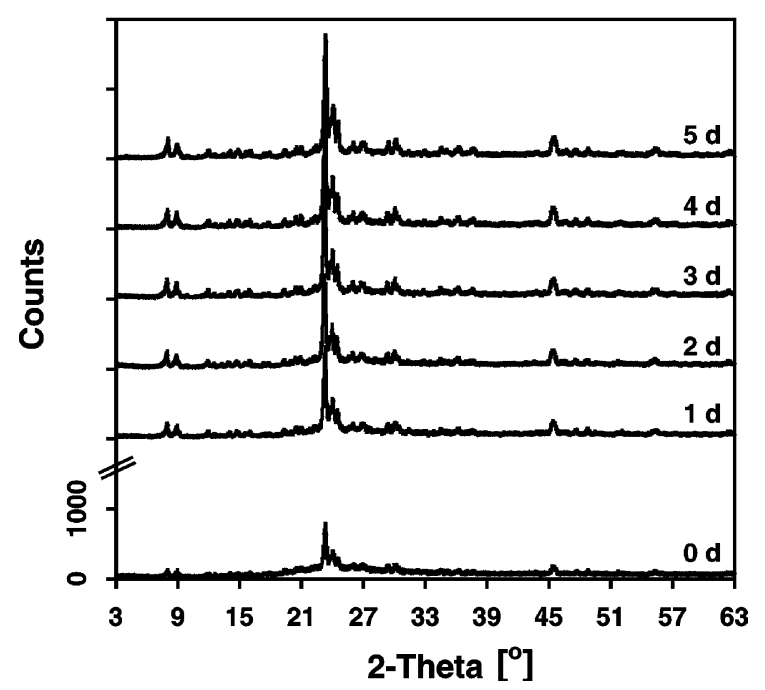

Fig. 5. XRD patterns of ZSM-5 synthesised in two stages with 33 wt. $\%$ silicalite-1 seeds; crystallisation times are indicated on the right side.

ticles in a narrow size distribution around $100 \mathrm{~nm}$ were not successful. In contrast, nano-particles can be produced very well in the Verduijn synthesis when aluminium is omitted. The particle size 


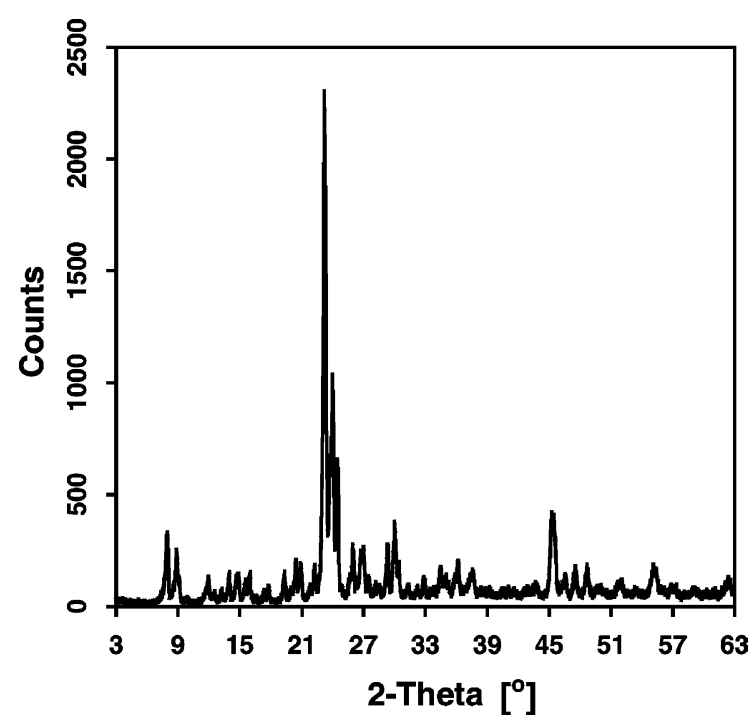

Fig. 6. XRD pattern of ZSM-5 synthesised after van Grieken [13]; crystallisation time: 2 days.

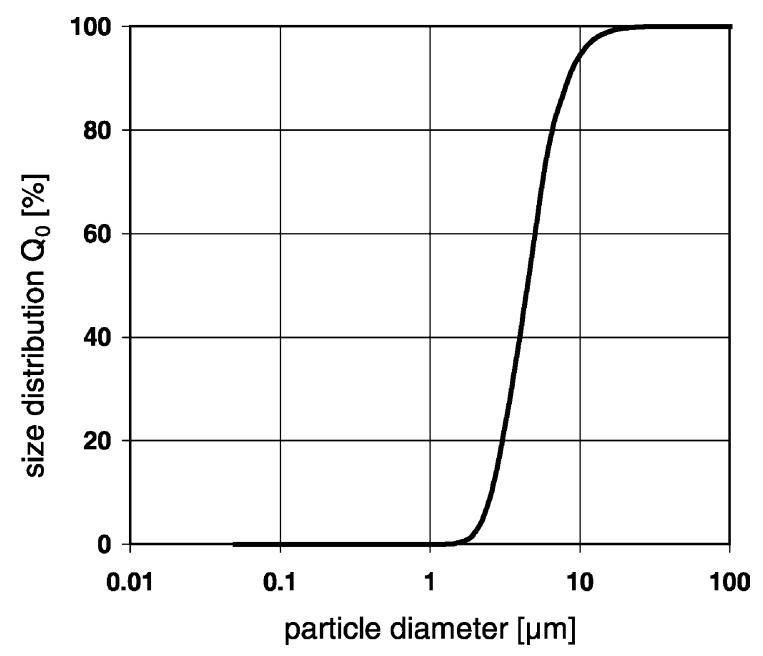

Fig. 7. Particle size distribution of ZSM-5 synthesised after Verduijn [4] in the presence of an aluminium source; crystallisation time: 12 days.

distribution of a silicalite- 1 obtained after $72 \mathrm{~h}$ is depicted in Fig. 9. Here, the size distribution is narrow. The mean particle size is $70 \mathrm{~nm}$, and more than $98 \%$ of the particles exhibit a diameter below $100 \mathrm{~nm}$.

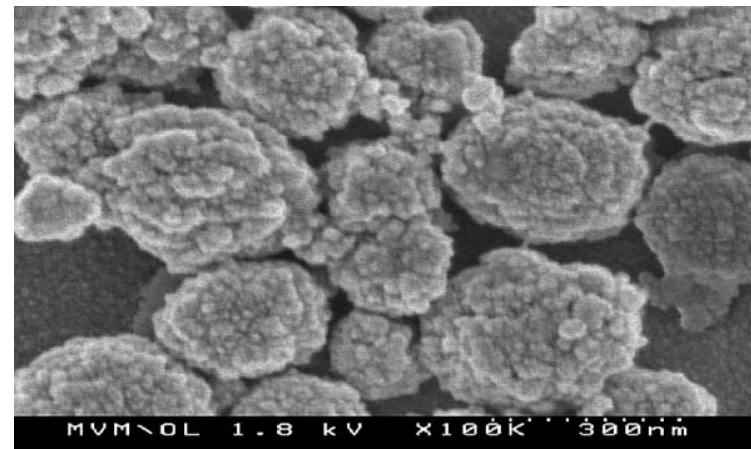

Fig. 8. SEM image of ZSM-5 synthesised after Verduijn [4] in the presence of an aluminium source; crystallisation time: 12 days.

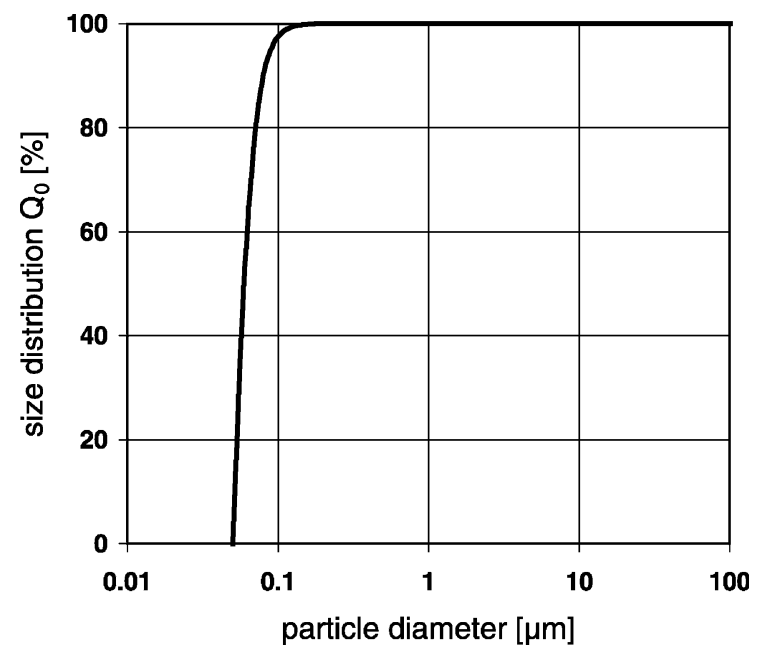

Fig. 9. Particle size distribution of silicalite-1 synthesised after Verduijn [4]; crystallisation time: 3 days.

When this material is employed for seeding aluminium-containing synthesis solutions, the particle size distributions of the products depend on the silicalite-1 concentration. This is shown in Fig. 10, where the size distributions of ZSM-5 after a crystallisation time of 8 days are plotted together. With the highest molar ratio of $a / b=9$ in the mixture (10 wt.\% silicalite-1 seeds, see experimental), a relatively low number of seed crystals can grow in a large reservoir of dissolved building units, and large particles are produced (Fig. 10b, right plot). A closer inspection of this material by 


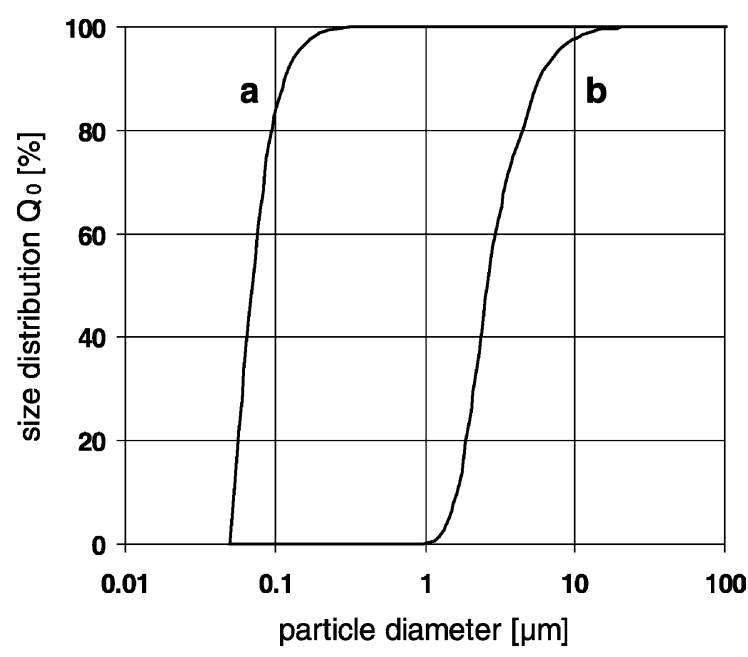

Fig. 10. Particle size distribution of ZSM-5 synthesised in two stages with silicalite- 1 seeds in a concentration of 33 and 23 wt. $\%$, identical curves (a), 10 wt. $\%$ (b); crystallisation time: 8 days in all cases.

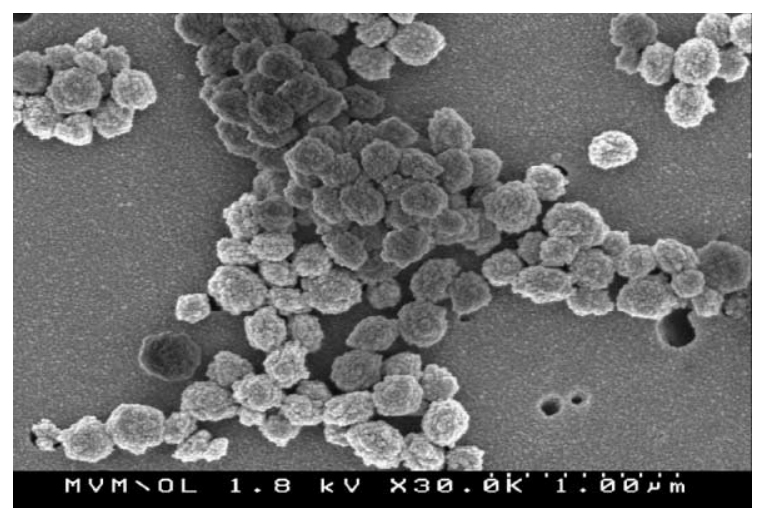

Fig. 11. SEM image of ZSM-5 synthesised in two stages with 10 wt. $\%$ silicalite-1 seeds; crystallisation time: 8 days.

means of SEM revealed, however, that the mean size of the individual particles amounted to about $0.3 \mu \mathrm{m}$, only (Fig. 11). The size distribution measurement by means of laser-Doppler anemometry gives a higher value because these particles stick together in lumps. The mechanical stability of the lumps is illustrated by the fact that they resisted the ultrasonic treatment prior to the measurement of the size distribution. In addition, the $\mathrm{pH}$ value of the suspensions $(\mathrm{pH}=10)$ was chosen such that repulsive electrostatic forces between the particles

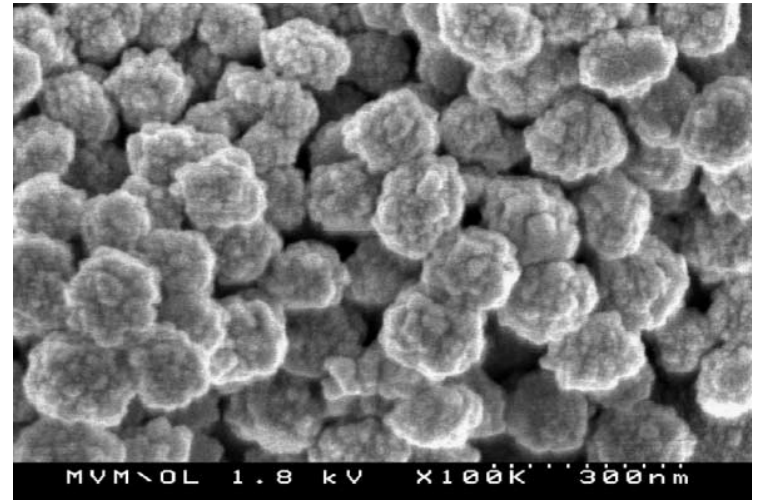

Fig. 12. SEM image of ZSM-5 synthesised in two stages with 33 wt.\% silicalite-1 seeds; crystallisation time: 8 days.

were at maximum. As shown in Fig. 10a (left plot), laser diffraction showed identical particle size distributions for the two synthesis mixtures with higher seed concentrations, i.e. $a / b=3.3$ and $a / b=2$. Again, a difference was observed in the corresponding SEM micrographs. Medium seed concentrations resulted in particles of $150 \mathrm{~nm}$, whereas the highest seed concentrations produced particles of $100 \mathrm{~nm}$ mean diameter. A SEM image of a product obtained with the highest seed concentration $(a / b=2)$ after a crystallisation time of 8 days is shown in Fig. 12. The particles exhibit an almost spherical shape. The rough surface and the cauliflower-like morphology are typical features of all samples crystallised in clear solution at atmospheric pressure. We have also investigated the effect of the crystallisation time on the crystal growth in the syntheses with seeds. However, mean particle sizes and size distributions changed during in the initial phase of the crystallisation, only. The length of the growth period decreased with increasing seed concentration and varied between 1 and 4 days.

The syntheses after van Grieken et al. yielded products with particle size distributions very close to those obtained with the seed-syntheses. This is suggested by the comparison of Fig. 13 with plot (a) in Fig. 10. A SEM image of a van Grieken-type ZSM-5 is depicted in Fig. 14. Here, single crystals are formed which exhibit smooth faces and edges. More detailed analyses of SEM micrographs 


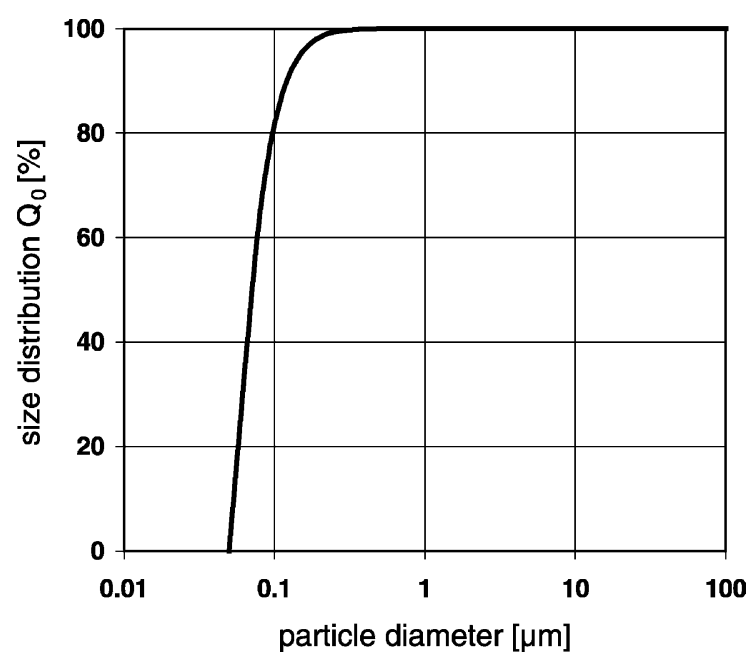

Fig. 13. Particle size distribution of ZSM-5 synthesised after van Grieken [13]; crystallisation time: 2 days.

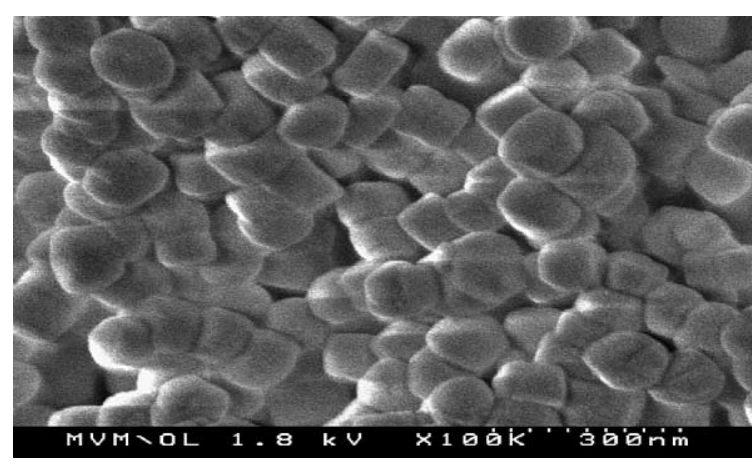

Fig. 14. SEM image of ZSM-5 synthesised after van Grieken [13]; crystallisation time: 2 days.

showed that the mean particle diameter amounted to about $90 \mathrm{~nm}$.

\subsection{Acidic properties}

The successful incorporation of aluminium into the framework and the possibility to produce nano-particles in the acidic $\mathrm{H}$-form was checked by means of temperature-programmed desorption of ammonia ( $\left.\mathrm{NH}_{3}-\mathrm{TPD}\right)$. XRD showed that the crystallinity of the samples was not affected by calcination and ion exchange.

Because ammonia was adsorbed at a relatively high temperature $(493 \mathrm{~K})$, the desorption spectra of all samples showed one peak, only, with a maximum at about $713 \mathrm{~K}$. Being aware of the semi-quantitative character of $\mathrm{NH}_{3}$-TPD measurements, we derived framework Si/Al ratios not from the absolute amounts of desorbed ammonia per unit mass of zeolite, but from relative magnitudes by taking reference samples of H-ZSM-5 of wellknown composition and "normal" crystal sizes between 0.5 and $3 \mu \mathrm{m}$ into account. The results of the TPD measurements are summarised in Table 1. They show that an Si/Al ratio of about 60 , which we aimed at, could be realised in all cases.

\section{Discussion}

With three out of four different synthesis methods under investigation it was possible to produce highly crystalline ZSM-5 zeolites. Focusing on nano-sized particles, however, only two of the methods were successful.

One of these involves hydrothermal crystallisation under autogenous pressure and has been developed by van Grieken et al. [13]. Typically, smooth single crystals are formed which, after a crystallisation time of $48 \mathrm{~h}$, exhibit a mean diameter of about $90 \mathrm{~nm}$. It is worth mentioning that we succeeded in reproducing this synthesis type many times and at first attempt. Some variations could be applied without problems which underlines the robustness of this approach.

The other method suitable to produce nanosized crystals involves crystallisation in open vessels at atmospheric pressure, however, in two subsequent steps. The first crystallisation is carried out in the absence of aluminium and yields very small silicalite-1 particles in a narrow size distribution. These are then used as seeds in the second crystallisation in the presence of an aluminium source. The over-all $\mathrm{Si} / \mathrm{Al}$ ratio of the products as measured by means of $\mathrm{NH}_{3}$-TPD matched very well the total Si/Al ratio in the synthesis mixture of the second crystallisation. This is remarkable because the Si/Al ratios of the clear synthesis solutions had to be varied in order to adjust the fixed ratio $\mathrm{Si} / \mathrm{Al}=60$ of the total mixture at varying seed concentrations. At the highest silicalite-1 concentration ( $33 \mathrm{wt} . \%$ of total silica content), for 
Table 1

Survey on the properties of selected synthesis products

\begin{tabular}{|c|c|c|c|c|c|c|}
\hline Method & Ref. & $\begin{array}{l}\mathrm{Si} / \mathrm{Al} \text { of synthesis } \\
\text { mixture }\end{array}$ & $\begin{array}{l}\text { Seeds } \\
\text { (wt. } \% \text { of silica) }\end{array}$ & $\begin{array}{l}\mathrm{D} \\
(\mathrm{nm})^{\mathrm{a}}\end{array}$ & $\begin{array}{l}\text { Crystals } \\
\text { agglomerated }\end{array}$ & $\begin{array}{l}\mathrm{Si} / \mathrm{Al} \text { of } \\
\text { product }^{\mathrm{b}}\end{array}$ \\
\hline Jacobsen & 14 & 60 & - & $-^{\mathrm{c}}$ & $-^{\mathrm{c}}$ & $-^{\mathrm{c}}$ \\
\hline van Grieken & 13 & 60 & - & 90 & No & $\approx 70$ \\
\hline Verduijn & $4^{\mathrm{d}}$ & 60 & - & $80-800^{e}$ & Yes & $\approx 70$ \\
\hline Verduijn & 4 & $\infty$ & - & 70 & No & $-($ Silicalite-1) \\
\hline Two-stage & This work & 60 & 10 & 300 & Yes & $\approx 50$ \\
\hline Two-stage & This work & 60 & 23 & 150 & No & $\approx 60$ \\
\hline Two-stage & This work & 60 & 33 & 100 & No & $\approx 60$ \\
\hline
\end{tabular}

${ }^{\mathrm{a}}$ Mean diameter as determined from SEM images.

${ }^{\mathrm{b}}$ Measured on $\mathrm{H}$-forms by means of $\mathrm{NH}_{3}$-TPD.

${ }^{\mathrm{c}}$ Products were amorphous.

${ }^{\mathrm{d}}$ With addition of an aluminium source; all examples in Ref. [4] describe syntheses without aluminium although the claims include ZSM-5.

${ }^{\mathrm{e}}$ Broad crystal size distribution.

instance, the $\mathrm{Si} / \mathrm{Al}$ ratios of the clear solutions employed were 40 . Obviously, the two-stage synthesis allows for a good control of the over-all $\mathrm{Si}$ / $\mathrm{Al}$ ratios of the products.

Unfortunately, we have no means to monitor the distribution of framework aluminium across such small crystals. It would be interesting to know whether the crystals consist of a silicalite-1 core and an aluminium-containing shell. In that case, the characteristic lengths for mass transport could be reduced even far below the $80-100 \mathrm{~nm}$ scale which is the typical size range of zeolite nanoparticles. Anyhow, the two-stage synthesis with silicalite-1 seeds has two interesting aspects. First, the particle size of the final product can be controlled very well via the concentration of the seeds in the synthesis mixture. Second, the static crystallisation at atmospheric pressure allows for simple scale-up at low equipment costs.

The two-stage approach has been derived from the methods developed by Verduijn and by Schoeman and Sterte known as "colloidal zeolite preparation". The direct, single-stage synthesis of colloidal aluminium-containing ZSM-5 according to this method failed, however. Broad particle size distributions were obtained and, in addition, the particles formed stable agglomerates in the size range of several micrometers. We cannot explain why aluminium has this negative effect on the particle size distribution when no seeds are present and will refrain from speculations. Our experimental methods do not provide sufficient infor- mation to draw conclusions about the mechanisms of crystallisation.

\section{Conclusions}

Two synthesis methods were identified which can be easily reproduced and yield disperse, nanocrystalline ZSM-5 with aluminium being incorporated into the framework. One of these, the synthesis after van Grieken et al., includes a single crystallisation under autogenous pressure. The other method was developed by ourselves and is based on two crystallisation steps under atmospheric pressure. The first is required to produce colloidal silicalite- 1 crystals which are then used as seeds in the second crystallisation. This approach appears to be cumbersome at first sight. However, the synthesis of the silicalite- 1 seeds is simple even on a larger scale, and previous studies have shown that aqueous sols of colloidal silicalite- 1 can be stabilised and stored for a long time [10]. In the two-stage synthesis of ZSM-5, the crystal size distribution of the final product can be controlled by choice of seed crystal concentration.

We suppose that the distribution of aluminium within the crystals is more or less homogeneous in the case of van Grieken-type products, whereas the two-stage synthesis results in egg-shell-type ZSM-5 crystals with aluminium being enriched in the shell. A more sophisticated characterisation of the particles will be required to examine this 
assumption. With both synthesis methods, however, it was possible to obtain products which could be transferred into the acidic $\mathrm{H}$-form. The over-all framework $\mathrm{Si} / \mathrm{Al}$ ratio of the products was found to be very close to the $\mathrm{Si} / \mathrm{Al}$ ratio of the synthesis mixture.

\section{Acknowledgement}

T. Mäurer thanks Max Buchner Forschungsstiftung for his grant.

\section{References}

[1] B. Kraushaar-Czarnetzki, J. Wijnbelt, WO 97/20016, to Shell Internationale Research Maatschappij, 1997.

[2] J.W. Gosselink, J.A.R. van Veen, WO 98/39096, to Shell Internationale Research Maatschappij, 1998.
[3] J.P. Verduijn, WO 93/08125, to Exxon Chemical Patents Inc., 1993.

[4] J.P. Verduijn, WO 97/03019, to Exxon Chemical Patents Inc., 1997.

[5] J.P. Verduijn, WO 97/03020, to Exxon Chemical Patents Inc., 1997.

[6] J.P. Verduijn, WO 97/03021, to Exxon Chemical Patents Inc., 1997.

[7] J.E. Otterstedt, P.J. Sterte, B.J. Schoeman, WO 94/05597, 1994.

[8] A.E. Persson, B.J. Schoeman, J. Sterte, J.E. Otterstedt, Zeolites 14 (1994) 557.

[9] B.J. Schoeman, J. Sterte, KONA 15 (1997) 150.

[10] T. Mäurer, S.P. Müller, B. Kraushaar-Czarnetzki, Ind. Eng. Chem. Res. 40 (2001) 2573.

[11] T. Mäurer, B. Kraushaar-Czarnetzki, Helv. Chim. Acta 84 (2001) 2550.

[12] B. Kraushaar-Czarnetzki, T. Mäurer, DE 10059 520, to Universität Karlsruhe (TH), 2001.

[13] R. van Grieken, J.L. Sotelo, J.L. Menendez, J.A. Melero, Micropor. Mesopor. Mater. 39 (2000) 135.

[14] C.J.H. Jacobsen, C. Madsen, T.V.W. Janssens, H.J. Jakobsen, J. Skibsted, Micropor. Mesopor. Mater. 39 (2000) 393. 